\title{
Erratum to: A phenomenological study of bottom-quark fragmentation in top-quark decay
}

\author{
Gennaro Corcella ${ }^{1,2,3, a}$, Federico Mescia ${ }^{4}$ \\ ${ }^{1}$ Museo Storico della Fisica e Centro Studi e Ricerche E. Fermi, Piazza del Viminale 1, 00184 Rome, Italy \\ ${ }^{2}$ Scuola Normale Superiore, Piazza dei Cavalieri 7, 56126 Pisa, Italy \\ ${ }^{3}$ Sezione di Pisa, INFN, Largo Fibonacci 3, 56127 Pisa, Italy \\ ${ }^{4}$ Departamento d'Estructura i Constituents de la Materia (ECM) and Institut de Ciencies del Cosmos (ICC), Universitat de Barcelona, \\ Av. Diagonal 647, 08028 Barcelona, Spain
}

Received: 24 May 2010 / Published online: 30 June 2010

(C) Springer-Verlag / Società Italiana di Fisica 2010

\section{Erratum to: Eur. Phys. J. C (2010) 65:171-180 DOI 10.1140/epjc/s10052-009-1170-4}

\begin{abstract}
We update the results on the $B$-lepton invariantmass distribution in the dilepton channel in top decay, with respect to the ones presented in Corcella and Mescia (Eur. Phys. J. C 65:171, 2010).
\end{abstract}

The figures and tables on the $m_{B \ell}$ invariant-mass distribution in top decay in [1] referred actually to the $p_{B} \cdot p_{\ell}$ scalar product. In the following, we shall present the correct figures and tables.

Figure 1 shows the $m_{B \ell}$ spectrum yielded by tuned and default HERWIG and PYTHIA, for a top mass value $m_{t}=$ $175 \mathrm{GeV}$. Figure 2 presents the invariant-mass distribution for $m_{t}=171$ and $179 \mathrm{GeV}$, according to HERWIG (a) and PYTHIA (b).

Tables 1 and 2 quote the first four Mellin moments of the $m_{B l}$ spectra, as given by the tuned versions of HERWIG and PYTHIA, respectively. The average value $\left\langle m_{B \ell}\right\rangle$ can be expressed in terms of $m_{t}$ according to a linear fit, by means of the least-square method, as follows:

$$
\begin{aligned}
& \left\langle m_{B \ell}\right\rangle_{\mathrm{H}} \simeq-25.31 \mathrm{GeV}+0.61 m_{t} ; \quad \delta=0.043 \mathrm{GeV}, \\
& \left\langle m_{B \ell}\right\rangle_{\mathrm{P}} \simeq-24.11 \mathrm{GeV}+0.59 m_{t} ; \quad \delta=0.022 \mathrm{GeV},
\end{aligned}
$$

The online version of the original article can be found under doi:10.1140/epjc/s10052-009-1170-4.

a e-mail: gennaro.corcella@sns.it where $\delta$ is the mean square deviation in the fit and the subscripts $\mathrm{H}$ and $\mathrm{P}$ refer to HERWIG and PYTHIA, respectively. The best-fit straight lines, as a function of $m_{t}$, are plotted in Fig. 3. As Tables 1 and 2 tell us that the typical difference between HERWIG and PYTHIA is $\left\langle m_{B \ell}\right\rangle \simeq$ $1.2-1.3 \mathrm{GeV}$, the corresponding uncertainty inferred on $m_{t}$ can be up to about $\Delta m_{t} \simeq 2 \mathrm{GeV}$, given the slopes of the straight lines in Fig. 3. If we discard the low- and high- $m_{B \ell}$ tails and restrict ourselves, e.g., to the range $50 \mathrm{GeV}<$ $m_{B \ell}<120 \mathrm{GeV}$, we obtain the truncated moments presented in Tables 3 and 4.

The best linear fits of the average value $\left\langle m_{B \ell}\right\rangle$ in terms of $m_{t}$ read:

$$
\begin{array}{ll}
\left\langle m_{B \ell}\right\rangle_{\mathrm{H}} \simeq 53.33 \mathrm{GeV}+0.18 m_{t} ; & \delta=0.034 \mathrm{GeV}, \\
\left\langle m_{B \ell}\right\rangle_{\mathrm{P}} \simeq 55.83 \mathrm{GeV}+0.17 m_{t} ; & \delta=0.020 \mathrm{GeV}
\end{array}
$$

The corresponding straight lines are plotted in Fig. 4. As the found discrepancy between HERWIG and PYTHIA is about $\Delta\left\langle m_{B \ell}\right\rangle \simeq 200-300 \mathrm{MeV}$, the induced uncertainty on the top mass, thinking of extracting it by fitting the mean value $\left\langle m_{B \ell}\right\rangle$, amounts to $\Delta m_{t} \simeq 1.5 \mathrm{GeV}$. The results on $\Delta m_{t}$ obtained in [1] are therefore confirmed.

Acknowledgement We are grateful to K. Melnikov, who pointed out an error in [1].

\section{References}

1. G. Corcella, F. Mescia, Eur. Phys. J. C 65, 171 (2010) 
Fig. $1 \quad B$-lepton invariant-mass distribution, in top decay and in the dilepton channel, according to tuned and default HERWIG and PYTHIA, for $m_{t}=175 \mathrm{GeV}$

Fig. $2 m_{B \ell}$ spectrum in top decay for $m_{t}=171$ and $179 \mathrm{GeV}$, according to default and tuned HERWIG (a) and PYTHIA (b)
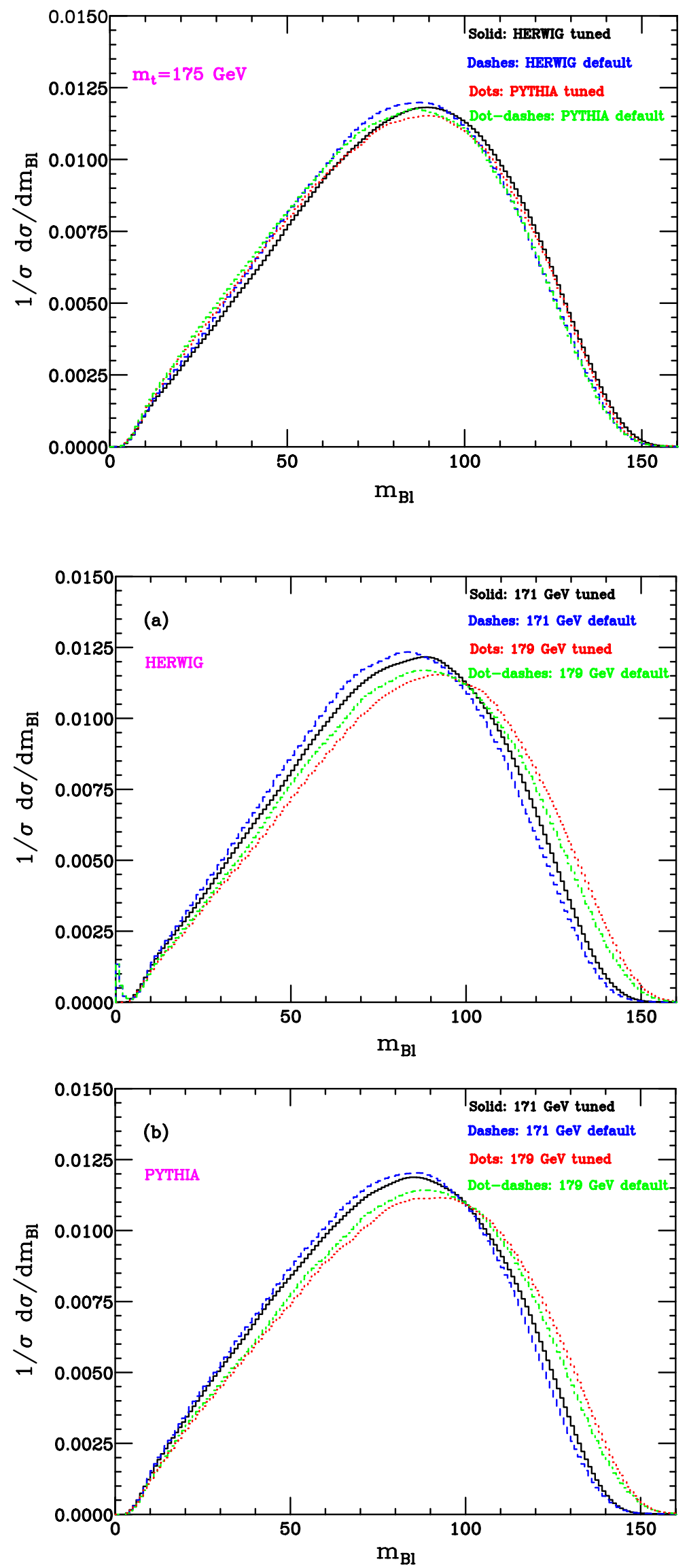
Table 1 First four moments of the $m_{B \ell}$ spectrum in top decay, yielded by HERWIG, after tuning the cluster model to ALEPH, OPAL and SLD data, for $171 \mathrm{GeV}<m_{t}<179 \mathrm{GeV}$

Table 2 As in Table 1, but using the PYTHIA event generator

Fig. 3 Linear fits of $\left\langle m_{B \ell}\right\rangle$ as a function of $m_{t}$, as obtained from HERWIG and PYTHIA codes

Table 3 Truncated moments of the $m_{B \ell}$ spectrum, according to HERWIG in the range $50 \mathrm{GeV}<m_{B \ell}<120 \mathrm{GeV}$

Table 4 As in Table 3, but using the PYTHIA event generator

\begin{tabular}{lllll}
\hline$m_{t}(\mathrm{GeV})$ & $\left\langle m_{B \ell}\right\rangle(\mathrm{GeV})$ & $\left\langle m_{B \ell}^{2}\right\rangle\left(\mathrm{GeV}^{2}\right)$ & $\left\langle m_{B \ell}^{3}\right\rangle\left(\mathrm{GeV}^{3}\right)$ & $\left\langle m_{B \ell}^{4}\right\rangle\left(\mathrm{GeV}^{4}\right)$ \\
\hline 171 & 78.39 & $7.01 \times 10^{3}$ & $6.82 \times 10^{5}$ & $7.02 \times 10^{8}$ \\
173 & 79.52 & $7.22 \times 10^{3}$ & $7.12 \times 10^{5}$ & $7.43 \times 10^{8}$ \\
175 & 80.82 & $7.45 \times 10^{3}$ & $7.46 \times 10^{5}$ & $7.91 \times 10^{8}$ \\
177 & 82.02 & $7.67 \times 10^{3}$ & $7.79 \times 10^{5}$ & $8.37 \times 10^{8}$ \\
179 & 83.21 & $7.89 \times 10^{3}$ & $8.13 \times 10^{5}$ & $8.86 \times 10^{8}$ \\
\hline
\end{tabular}

\begin{tabular}{lllll}
\hline$m_{t}(\mathrm{GeV})$ & $\left\langle m_{B \ell}\right\rangle(\mathrm{GeV})$ & $\left\langle m_{B \ell}^{2}\right\rangle\left(\mathrm{GeV}^{2}\right)$ & $\left\langle m_{B \ell}^{3}\right\rangle\left(\mathrm{GeV}^{3}\right)$ & $\left\langle m_{B \ell}^{4}\right\rangle\left(\mathrm{GeV}^{4}\right)$ \\
\hline 171 & 77.17 & $6.85 \times 10^{3}$ & $6.62 \times 10^{5}$ & $6.81 \times 10^{8}$ \\
173 & 78.37 & $7.06 \times 10^{3}$ & $6.94 \times 10^{5}$ & $7.23 \times 10^{8}$ \\
175 & 79.55 & $7.27 \times 10^{3}$ & $7.25 \times 10^{5}$ & $7.67 \times 10^{8}$ \\
177 & 80.70 & $7.48 \times 10^{3}$ & $7.56 \times 10^{5}$ & $8.12 \times 10^{8}$ \\
179 & 81.93 & $7.71 \times 10^{3}$ & $7.91 \times 10^{5}$ & $8.61 \times 10^{8}$ \\
\hline
\end{tabular}

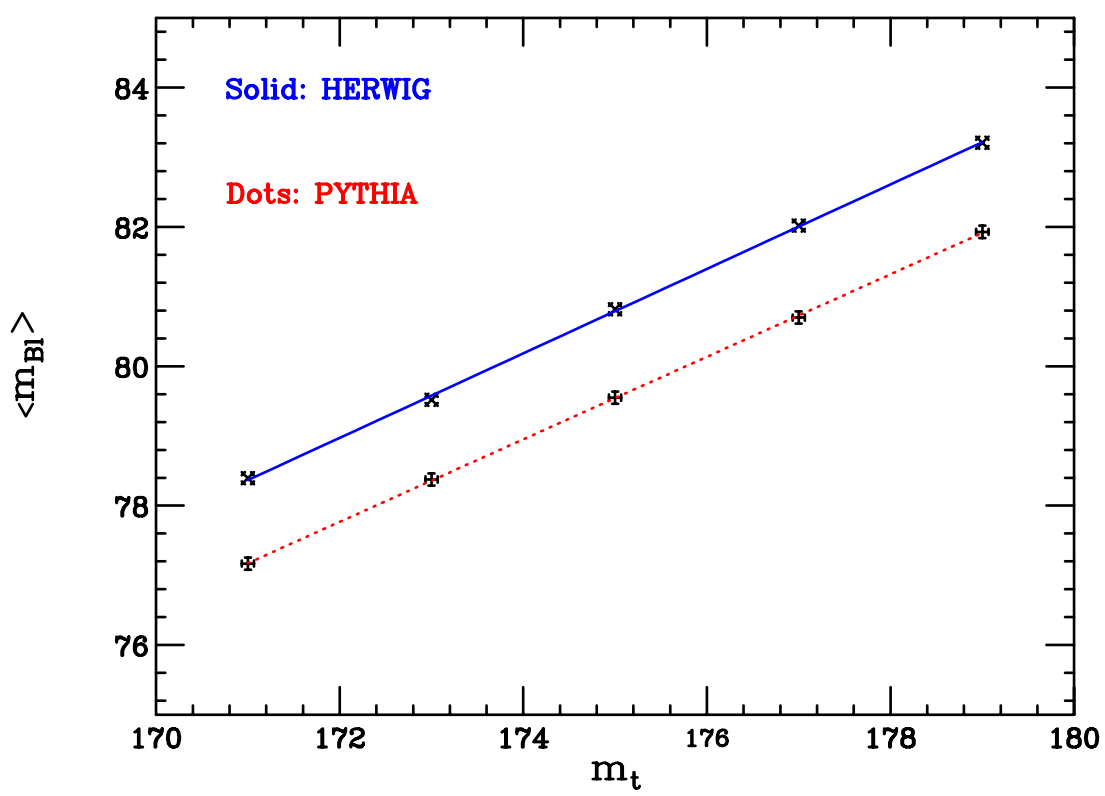

\begin{tabular}{lllll}
\hline$m_{t}(\mathrm{GeV})$ & $\left\langle m_{B \ell}\right\rangle(\mathrm{GeV})$ & $\left\langle m_{B \ell}^{2}\right\rangle\left(\mathrm{GeV}^{2}\right)$ & $\left\langle m_{B \ell}^{3}\right\rangle\left(\mathrm{GeV}^{3}\right)$ & $\left\langle m_{B \ell}^{4}\right\rangle\left(\mathrm{GeV}^{4}\right)$ \\
\hline 171 & 84.64 & $7.52 \times 10^{3}$ & $6.97 \times 10^{5}$ & $6.70 \times 10^{8}$ \\
173 & 85.01 & $7.59 \times 10^{3}$ & $7.06 \times 10^{5}$ & $6.81 \times 10^{8}$ \\
175 & 85.43 & $7.66 \times 10^{3}$ & $7.17 \times 10^{5}$ & $6.94 \times 10^{8}$ \\
177 & 85.78 & $7.72 \times 10^{3}$ & $7.25 \times 10^{5}$ & $7.04 \times 10^{8}$ \\
179 & 86.09 & $7.78 \times 10^{3}$ & $7.32 \times 10^{5}$ & $7.13 \times 10^{8}$ \\
\hline
\end{tabular}

\begin{tabular}{lllll}
\hline$m_{t}(\mathrm{GeV})$ & $\left\langle m_{B \ell}\right\rangle(\mathrm{GeV})$ & $\left\langle m_{B \ell}^{2}\right\rangle\left(\mathrm{GeV}^{2}\right)$ & $\left\langle m_{B \ell}^{3}\right\rangle\left(\mathrm{GeV}^{3}\right)$ & $\left\langle m_{B \ell}^{4}\right\rangle\left(\mathrm{GeV}^{4}\right)$ \\
\hline 171 & 84.42 & $7.49 \times 10^{3}$ & $6.93 \times 10^{5}$ & $6.65 \times 10^{8}$ \\
173 & 84.79 & $7.55 \times 10^{3}$ & $7.02 \times 10^{5}$ & $6.77 \times 10^{8}$ \\
175 & 85.13 & $7.61 \times 10^{3}$ & $7.10 \times 10^{5}$ & $6.87 \times 10^{8}$ \\
177 & 85.45 & $7.67 \times 10^{3}$ & $7.18 \times 10^{5}$ & $6.97 \times 10^{8}$ \\
179 & 85.77 & $7.73 \times 10^{3}$ & $7.26 \times 10^{5}$ & $7.06 \times 10^{8}$ \\
\hline
\end{tabular}


Fig. 4 Linear fits of $\left\langle m_{B \ell}\right\rangle$, as a function of $m_{t}$, using HERWIG and PYTHIA in the range

$50 \mathrm{GeV}<m_{B \ell}<120 \mathrm{GeV}$

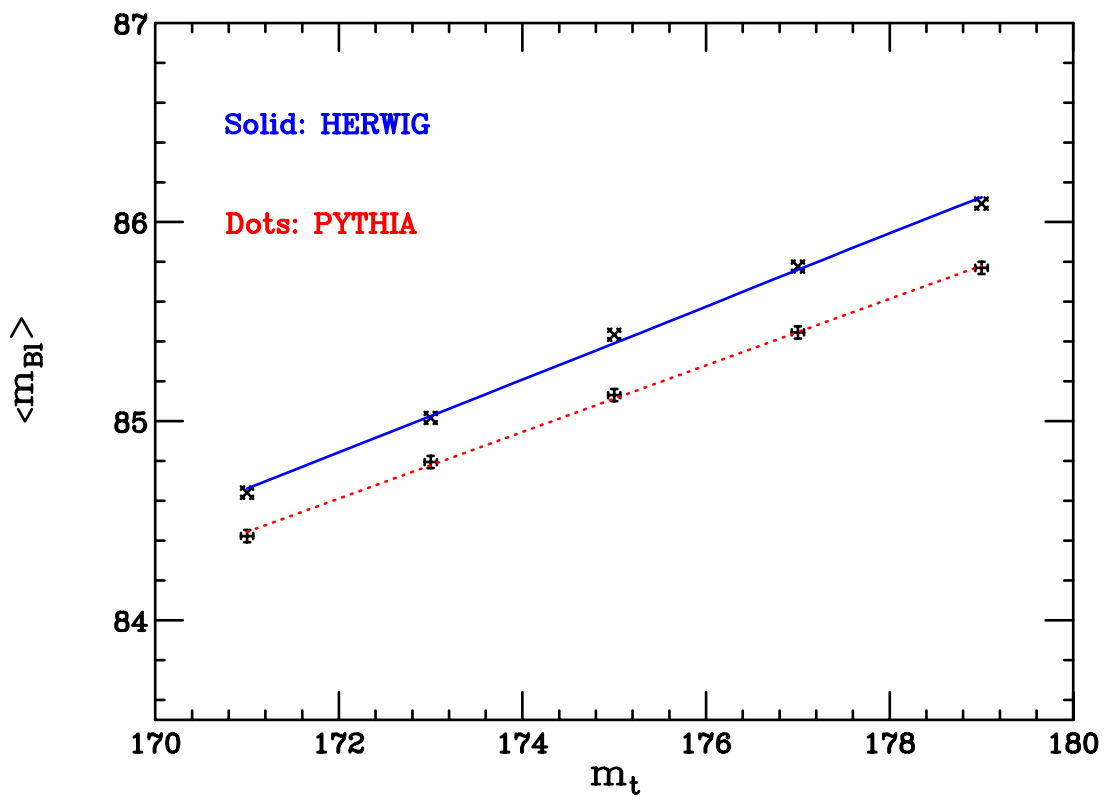

